\title{
Status of ART-XC / SRG instrument
}

M. Pavlinsky ${ }^{1}$, V. Akimov ${ }^{1}$, V. Levin ${ }^{1}$, I. Lapshov ${ }^{1}$, A. Tkachenko ${ }^{1}$, N. Semena ${ }^{1}$, M. Buntov ${ }^{1}$, A. Glushenko', V. Arefiev ${ }^{1}$, A. Yaskovich ${ }^{1}$, R. Sunyaev ${ }^{1,4}$, E. Churazov ${ }^{1,4}$, M. Gilfanov ${ }^{1,4}$, S. Grebenev ${ }^{1}$, S. Sazonov ${ }^{1}$, M. Revnivtsev ${ }^{1}$, A. Lutovinov ${ }^{1}$, S. Molkov ${ }^{1}$, M. Kudelin ${ }^{1}$, T. Drozdova ${ }^{1}$, S. Garanin ${ }^{2}$, S. Grigorovich ${ }^{2}$, D. Litvin ${ }^{2}$, V. Lazarchuk ${ }^{2}$, I. Roiz ${ }^{2}$, M. Garin ${ }^{2}$, V. Babyshkin ${ }^{3}$, I. Lomakin ${ }^{3}$, A. Menderov ${ }^{3}$, D. Moskvinov ${ }^{3}$, M. Gubarev ${ }^{5}$, B. Ramsey ${ }^{5}$, K. Kilaru ${ }^{5}$, S.L. O’Dell ${ }^{5}$, J. Kolodziejczak ${ }^{5}$, R. Elsner ${ }^{5}$

${ }^{1}$ Space Research Institute, Russia

${ }^{2}$ The All-Russian Scientific Research Institute for Experimental Physics, VNIIEF, Russia

${ }^{3}$ Lavochkin Association, Russia

${ }^{4}$ MPI für Astrophysik, Germany

${ }^{5}$ NASA Marshall Space Flight Ctr., USA

\begin{abstract}
Spectrum Roentgen Gamma (SRG) is an X-ray astrophysical observatory, developed by Russia in collaboration with Germany. The mission will be launched in March 2016 from Baikonur, by a Zenit rocket with a Fregat booster and placed in a 6-month-period halo orbit around L2. The scientific payload consists of two independent telescopes - a softX-ray survey instrument, eROSITA, being provided by Germany and a medium-X-ray-energy survey instrument ARTXC being developed by Russia. ART-XC will consist of seven independent, but co-aligned, telescope modules. The NASA Marshall Space Flight Center (MSFC) is fabricating the flight mirror modules for the ART-XC/SRG. Each mirror module will be aligned with a focal plane CdTe double-sided strip detectors which will operate over the energy range of 6-30 keV, with an angular resolution of $<1^{\prime}$, a field of view of $\sim 34^{\prime}$ and the expected energy resolution of about $10 \%$ at $14 \mathrm{keV}$.
\end{abstract}

\section{SRG Overview}

The Spectrum-Roentgen-Gamma (SRG) Mission is a Russian - German X-ray astrophysical observatory. A schematic representation of the SRG satellite is shown in Figure 1. The Max-Planck-Institut für Extraterrestrische Physik (MPE), Germany, is responsible for the development of the first mission instrument - the X-ray grazing-incidence mirror telescope extended ROentgen Survey with an Imaging Telescope Array (eROSITA) [1]. The second instrument is the Astronomical Roentgen Telescope - X-ray Concentrator (ART-XC) [2], an X-ray mirror telescope with a harder response than eROSITA. The ART-XC instrument is being developed by the Russian Space Research Institute (IKI) and the All-Russian Scientific Research Institute for Experimental Physics (VNIIEF). The NASA Marshall Space Flight Center (MSFC) is fabricating the flight $\mathrm{x}$-ray optics for the ART-XC instrument.

The scientific payload is housed on the Navigator platform, developed by Lavochkin Association (Russia). Such platforms are now in service on the Elektro-L mission (January 2011 - April 2014) and the Spektr-R mission (since July 2011). The details of the platform design parameters are presented in Table 1. The Navigator platform has been developed to be a universal medium-class platform for scientific missions planned to be launched to different orbits. The platform has been designed for autonomous operation of scientific payloads and can provide 3-axis stabilization or rotation with variable speed - an important feature to be utilized by the SRG payload during its mission.

Spectrum-RG will be launched in March 2016 from Baikonur and delivered to L2 point with use of the Zenit-2SB rocket and Fregat-SB booster. 
The SRG observing program is divided into three stages over the 7.5-year mission lifetime. The first $\sim 100$ days during the transit to the L2 point will be devoted to initial check-up and in-flight calibrations. The next 4 years will be devoted to the all-sky survey. During this time, the SRG observatory will rotate around the axis pointed approximately between the Sun and the Earth, with a period of about four hours. The final stage of the mission, which will last about 3 years,

| will be spent on pointed observations of selected objects from the most interesting galaxy clusters, AGNs and galaxy sources. Short (up to one month) pointed observations are possible after each half-year survey period.

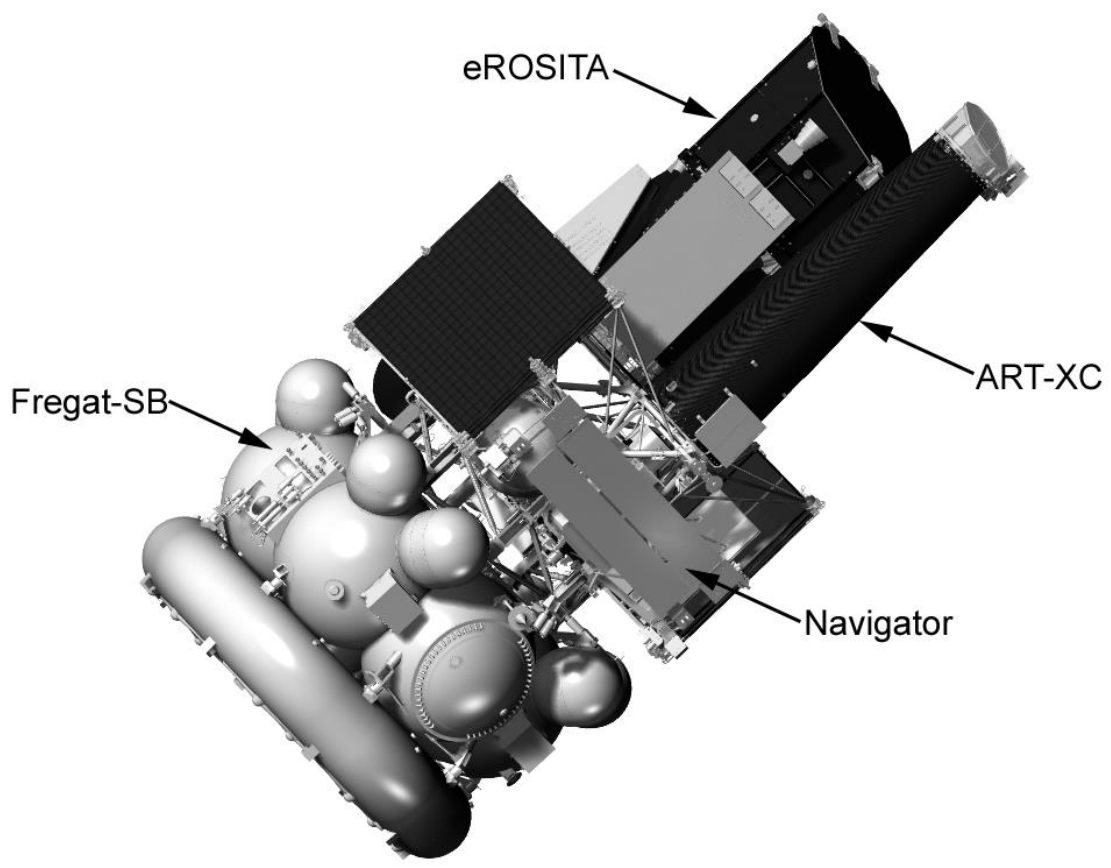

Figure 1. The SRG satellite utilizes the Russian Navigator platform. The eROSITA and the ART-XC telescopes, installed on the platform, will be launched from Baikonur and delivered to L2 point with use of the Zenit-2SB rocket and Fregat-SB booster.

Table 1. Key parameters of the Spectrum-Roentgen-Gamma mission

\begin{tabular}{|l|l|}
\hline Launch date & March 2016 \\
\hline Launch site & Baikonur \\
\hline Space Transportation technologies & $\begin{array}{l}\text { "Zenit-2SB" LV, } \\
\text { "Fregat-SB" upper stage }\end{array}$ \\
\hline Operational orbit & Region of libration point L2 \\
\hline Active lifetime & 7.5 years \\
\hline S/C dry mass & $2267 \mathrm{~kg}$ \\
\hline Payload mass & $1228 \mathrm{~kg}$ \\
\hline S/C wet mass & $2647 \mathrm{~kg}$ \\
\hline Radio line frequency range & $\mathrm{X} \mathrm{band}$ \\
\hline Science Data Transmission Rate & $512 \mathrm{kbit} / \mathrm{sec}$ \\
\hline Payload power consumption & $680 \mathrm{~W}$ \\
\hline
\end{tabular}


The mission will conduct an all-sky survey in the $0.5-11 \mathrm{keV}$ band with the imaging telescopes eROSITA and ART-XC. It will permit the discovery of all obscured accreting Black Holes in nearby galaxies, many ( millions) new distant AGN, and the detection of all massive clusters of galaxies in the Universe. In addition to the all-sky survey, dedicated sky regions will be observed with higher sensitivity and thereafter follow-on pointed observations of selected sources at energies up to $30 \mathrm{keV}$ will take place in order to investigate the nature of dark matter, dark energy and physics of accretion.

\section{2. $\quad$ ART-XC}

\subsection{Instrument description}

The ART-XC instrument consists of seven co-aligned X-ray mirror modules coupled with seven CdTe doubled sided strip detectors as shown schematically in Figure 2. The mirror modules are installed on an optical bench plate mounted on the top of a carbon fiber optical bench. Each x-ray mirror module is focused onto its own detector and thus seven detectors are installed on the bottom of the optical bench. Table 2 summarizes the overall ART-XC instrument performance specifications.

Table 2. Key parameters of ART-XC.

\begin{tabular}{|l|c|}
\hline Energy range & $6-30 \mathrm{keV}$ \\
\hline Field of view & $\varnothing 34^{\prime}$ \\
\hline On-axis angular resolution & $<1^{\prime}$ \\
\hline Energy resolution & $10 \%$ at $14 \mathrm{keV}$ \\
\hline Effective area for pointed observations & $450 \mathrm{~cm}^{2} @ 8 \mathrm{keV}$ \\
\hline Grasp for survey & $>40 \mathrm{deg}^{2} \mathrm{~cm}^{2} @ 8 \mathrm{keV}$ \\
\hline Time resolution & $1 \mathrm{~ms}$ \\
\hline
\end{tabular}

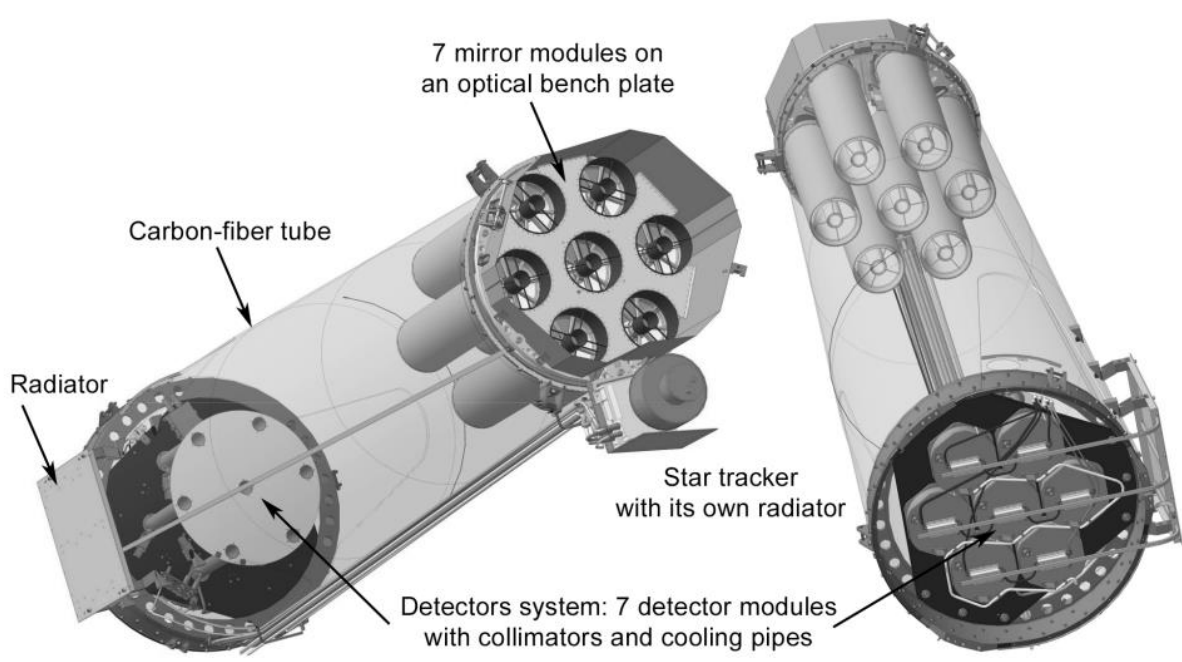

Formatted: English (U.S.)

Figure 2. The ART-XC telescope with seven mirror modules and seven focal-plane detectors. The basic structure of ART-XC is the optical bench - the conical carbon-fiber tube which is equipped with a moveable cover to protect the optics during launch (not shown).The star tracker with its own radiator is installed on the top of this tube. The detector collimators, cooling radiator with cooling pipes and the electronics boxes are visible at the bottom of the tube. 

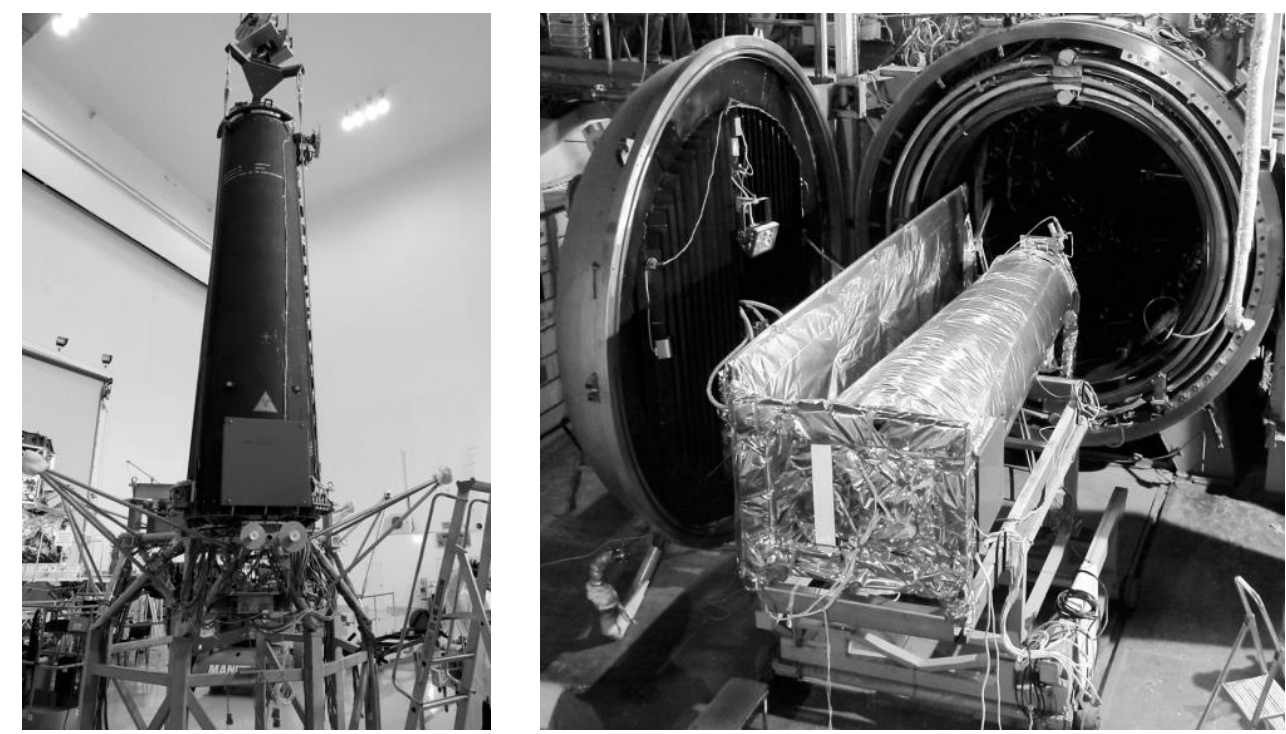

Figure 3. The left panel shows the engineering model (EM) of the ART-XC. The fFirst run of EM ART-XC- S/C electrical tests was completed March 2014. Right panel shows the Structural-Thermo Model (STM) before placing it into the $60 \mathrm{~m}^{3}$ vacuum chamber.

The autonomous, mechanical and thermo-vacuum tests of the ART-XC Structural-Thermo Model (STM) were completed in early 2012. More detailed information of the STM ART-XC thermal-vacuum are presented in Semena, et al. "ART-

XC/SRG: Results of thermo-vacuum tests" in the proceeding of this conference. The Ffirst run of the EM ART-XC- S/C electrical tests was completed March 2014 and= iIn June 2014 the qualification test of the ART-XC subsystems will be completed. A Đdisassembled electrical test is scheduled forin July 2014. Assembly ofing the ART-XC qualification model (QM) will start in August 2014 and- tThe delivery date for the flight model (FM) of the ART-XC instrument in the Lavochkin Association is summer 2015.

\subsection{The optical system}

A schematic representation of the ART-XC mirror system is shown in the left panel-Figure 4. Each x-ray mirror module contains 28 mirror shells fabricated using an electroformednickel-replication technique. The shells are glued in to a supporting spider and the spider is installed onto the optical plate of the instrument. The shell diameters vary from $\sim 50$ to $150 \mathrm{~mm}$ and the focal distance of the mirror system is $2700 \mathrm{~mm}$. In order to extend the energy response to $30 \mathrm{keV}$ the mirror inner surface will be coated with $10-20 \mathrm{~nm}$ of iridium. The required system angular resolution of better than 1 arc minute necessitates that the shells be figured to a Wolter 1 prescription.

The weight limit for the ART-XC module is $17 \mathrm{~kg}$. The shell thickness varies slightly with radius so the outer shells have larger than nominal thickness to make them stiffer and, hence, to improve the angular resolution of a module.

The NASA Marshall Space Flight Center (MSFC) is fabricating | the -all seven flight mirror modules for the ART-XC/SRG. Four

Table 3. Summary of the mirror system design

\begin{tabular}{|l|c|}
\hline Number of mirror systems & 7 \\
\hline Number of nested mirror shells & 28 \\
\hline Form of shell & Wolter-I \\
\hline On-axis angular resolution, HPD & $<40^{\prime \prime}$ \\
\hline Focal length & $2700 \mathrm{~mm}$ \\
\hline Length of shell & $580 \mathrm{~mm}$ \\
\hline $\begin{array}{l}\text { Diameters of mirror shells } \\
\text { (intersection) }\end{array}$ & $49-145 \mathrm{~mm}$ \\
\hline Material of shells & Ni/Co \\
\hline Mirror coating materials & Iridium \\
\hline
\end{tabular}


| of them are being fabricateding under a Reimbursable Agreement between NASA and IKI and will be delivered in September 2014. Up to date these four ART-XC flight mirror modules have been assembled and two of them passed the vibration tests and have been calibrated at MSFC's 104-m Stray Light Facility. The remaining three flight modules and | an additional spare unit are being fabricated under a Cooperative Agreement between NASA and IKI. The preliminary date of delivery for these modules is October 2014. More detailed information of the ART-XC mirror modules status and results of their calibration are presented in Gubarev, et al. "ART-XC/SRG: Status of the X-ray optics development" and Gubarev, et al. "Calibration of the ART-XC/SRG x-ray mirror modules" in the proceeding of this conference.

The on--axis effective area of each ART-XC mirror module should be greater than $\sim 65 \mathrm{~cm}^{2}$ at $8 \mathrm{keV}$. Figure 5 shows the measured on--axis effective area of one ART-XC mirror module as a function of X-ray energy and the expected effective area $\left(A_{e f f}\right)$ at $8 \mathrm{keV}$ at the nominal focus for an off-axis source at infinite distance. The diameter of the detector is $28.56 \mathrm{~mm}$. The detector is placed at the nominal focus and is assumed to have $100 \%$ efficiency. The different curves | represent the effective areas for the rays doubly (D) reflected from the both parabolic and hyperbolic mirrors, for the rays singly reflected from the parabolic $(\mathrm{P})$ or hyperbolic $(\mathrm{H})$ mirrors, and for unreflected rays $(\mathrm{S})$ passing straight-through the mirror module without any reflection.

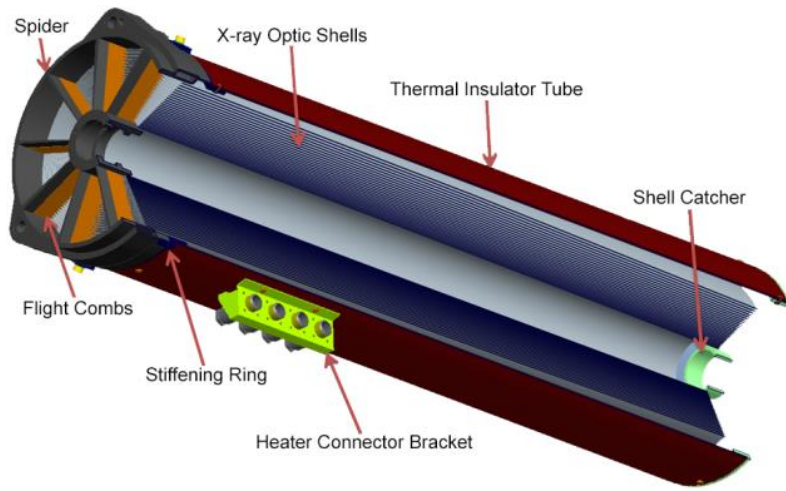

Figure 4. A cross section of an ART-XC X-ray mirror module. An inner baffle tube (for stray light reduction) and the module heaters are not shown.
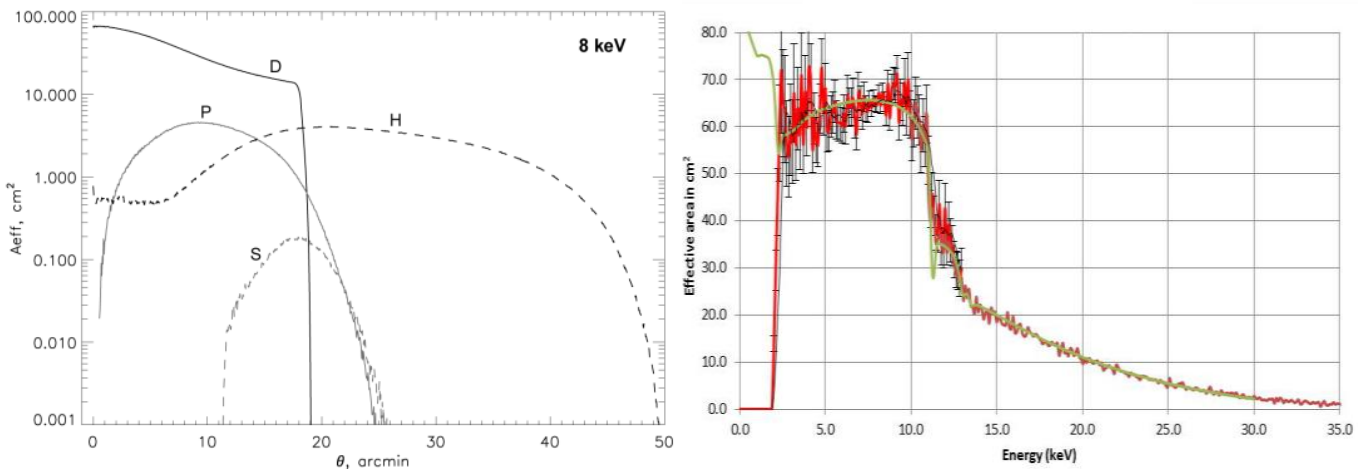

Figure 5. The left panel shows the simulated effective area of thefor one ART-XC mirror module, $A_{\text {eff }}$ in $\mathrm{cm}^{2}$, at $8 \mathrm{keV}$ vs. polar offaxis angle $\theta$ in arcmin. The diameter of the detector is $28.56 \mathrm{~mm}$. The detector is placed at the nominal focus and is assumed to have $100 \%$ efficiency. The different curves are rays doubly reflected from the $\mathrm{P}$ and $\mathrm{H}$ mirrors (D, black solid curve), rays singly reflected from the parabolic mirrors ( $\mathrm{P}$, gray solid curve), rays singly reflected from the hyperbolic mirrors $(\mathrm{H}$, black dashed curve), and | unreflected rays passing straight-through the mirror module ( $\mathrm{S}$, gray dashed curve). The right panel shows the measured on--axis effective area of an ART-XC mirror module as a function of X-ray energy. More detailed results of the ART-XC module calibration are presented in Gubarev, et al. "Calibration of the ART-XC/SRG x-ray mirror modules" in the proceeding of this conference. 


\subsection{Focal plane detector system}

Each mirror module is aligned with a focal plane detector. The detector system of ART-XC consist of seven X-ray | detector bloksmodules, two electronic modulesbloks and serial interface connection moduleblock. Developed by IKI, the ART-XC focal plane uses cadmium-telluride (CdTe) Schottky Diode double-sided strip detectors, read by custom Application Specific Integrated Circuits (ASICs). The high quality CdTe crystals were manufactured by Acrorad Co.

| Ltd. (Japan). Each detector has 48 strips on the top and 48 strips on the bottom layer of the CdTe die crystal on a $595 \mu \mathrm{m}$ pitch giving a field of view diameter of about 36 arcmin. Combining the detector's spatial resolution with the mirror module's angular resolution results in an expected system resolution of around 1 arcmin. Table 4 shows the ART-XC detector parameters.

| The VA64TA1 ASICs is used as the front-end electronic for the CdTe detector. The measured noise of the VA64TA1 spectrometric channels without detector is $0.69 \mathrm{keV}$ (FWHM) or $70 \mathrm{e}^{-}$(RMS). A low-energy threshold of $5 \mathrm{keV}$ is now being achieved in flight development units. The measured average energy resolution of the bottom layer of a QM ART$\mathrm{XC}$ detector is $1.7 \mathrm{keV}$ at $13.9 \mathrm{keV}$. The average energy resolution of the top is $2.2 \mathrm{keV}$ at $13.9 \mathrm{keV}$. It is expected that the energy resolution of an ART-XC detector can be improved by $10 \%$ at $14 \mathrm{keV}$ when the sum of the signals from the

bottom and the top layers to be used. Figure 6 shows the spectra of ${ }^{55} \mathrm{Fe}$ and ${ }^{241} \mathrm{Am}$ sources for bottom and top layers of a QM ART-XC detector. The images obtained with one of the QM ART-XC detector in three energy bands 2.5-7.5 keV $\left({ }^{55} \mathrm{Fe} 5.95 \mathrm{keV}\right), 10-28 \mathrm{keV}\left({ }^{237} \mathrm{~Np}\right.$ lines: $11.89,13.9,17.81$ and $\left.20.82 \mathrm{keV} ;{ }^{241} \mathrm{Am} 26.34 \mathrm{keV}\right)$ and $54-61 \mathrm{keV}\left({ }^{241} \mathrm{Am}\right.$ $59.6 \mathrm{keV}$ ) are shown in the Figure 7. The dark areas on these images are a shadow from the cover of the detector vacuum-tight housing with beryllium entrance window of $30 \mathrm{~mm}$ diameter. The detector cover is transparent for the photons at energies higher than $50 \mathrm{keV}$, which is clearly seen on the right panel of this figure.

To reduce polarization effects, the detector crystal is cooled to $-30^{\circ} \mathrm{C}$ in space. A thin $(100 \mu \mathrm{m})$ beryllium window of | diameter $30 \mathrm{~mm}$ seals a vacuum-tight housing that prevents condensation onto the crystal surface during ground tests. The ground tests utilize a built-in Peltier cooler; flight operations will use a passive system of thermal tubing and an external radiator. The evolution of ${ }^{55} \mathrm{Fe}$ and ${ }^{241} \mathrm{Am}$ spectrum as function of time for bottom and top layers is shown in Figure 8. Every vertical line is the sum of spectra obtained in all 48 strips. The spectra are stable in both layers of the ART-XC detector up to 67 hours at $-29^{\circ} \mathrm{C}$ and $-100 \mathrm{~V}$ bias. At $+10^{\circ} \mathrm{C}$, the detector will work without spectral degradation ef spectra for about one hour.

A cylindrical collimator of height $380 \mathrm{~mm}$ and inside diameter $40 \mathrm{~mm}$ limits the diffuse background reaching the detector. This collimator is fitted with graded shielding: the bottom half with tin, copper, and aluminum; the top half with just copper and aluminum. The collimator base incorporates the block of calibration sources $\left({ }^{55} \mathrm{Fe}+{ }^{241} \mathrm{Am}\right)$ for in-flight calibration.

| Up to date seven qualification models $(\mathrm{QM})$ of the ART-XC x-ray detector blocks have been assembled. The detectors were degreased and varnished and successfully passed the vibration tests and the technological run at room temperature as well as at high $\left(+55^{\circ} \mathrm{C}\right)$ and low $\left(-35^{\circ} \mathrm{C}\right)$ temperatures (see Figure 9 and 10). The approval, acceptance and qualification tests of the QM x-ray detector blocks will be held in June 2014.
Comment [BDR1]: Not sure what 'block' me here. If it is a single unit for each 'block' then uni module is a better word. If it is a system, then sys may be a better word.

Comment [BDR2]: Crystal may be a better w here

Comment [BDR3]: Table 2 gives 34 arcmin

Comment [BDR4]: Improved by $10 \%$ or improved to $10 \%$. I think $10 \%$ is the final value.
Comment [BDR5]: Not sure what this mean Conformal coatings of electronics, or surface treatment of housings? 
URD05, DSSD CdTe, bottom, $\mathrm{Fe}^{55}+\mathrm{Am}^{241}, \mathrm{~T}=-25.6 \mathrm{deg} \mathrm{C}, \mathrm{U}=-100 \mathrm{~V}$

URD05, DSSD CdTe, top, $\mathrm{Fe}^{55}+\mathrm{Am}^{241}, \mathrm{~T}=-25.6 \mathrm{deg} \mathrm{C}, \mathrm{U}=-100 \mathrm{~V}$
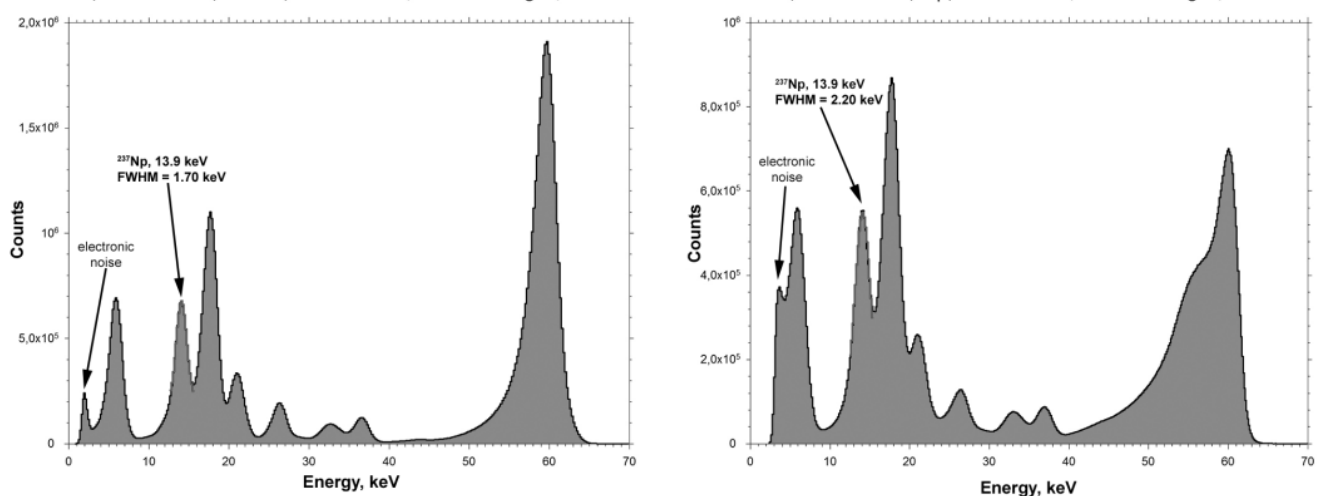

Figure 6. Energy spectrum of ${ }^{55} \mathrm{Fe}$ and ${ }^{241} \mathrm{Am}$ sources obtained from the bottom (left) and top (right) layers of a QM detector.
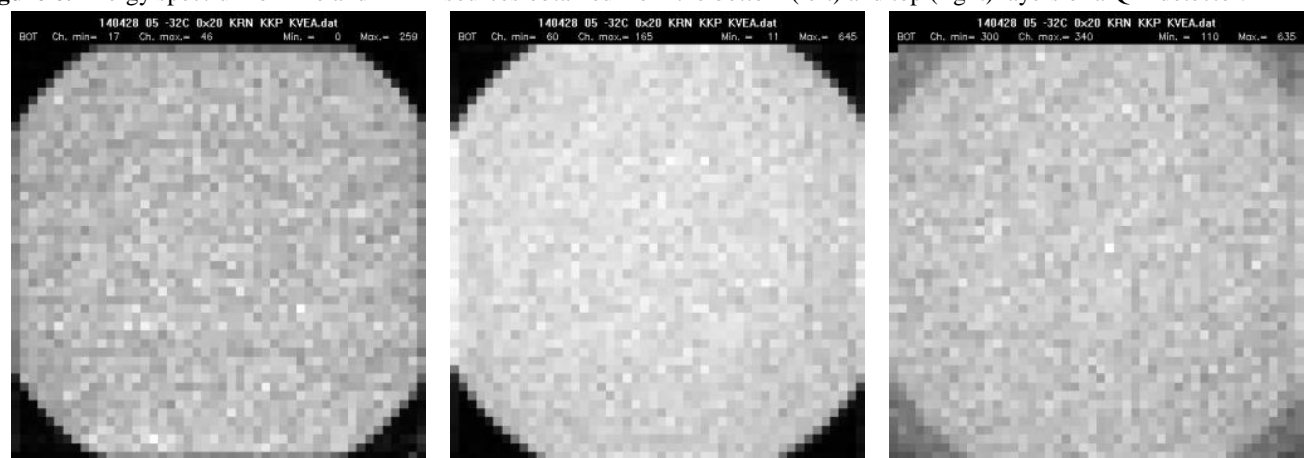

Figure 7. The images of ${ }^{55} \mathrm{Fe}+{ }^{241} \mathrm{Am}$ sources obtained from one of the QM ART-XC detector in the three energy bands. The left panel shows the image at $2.5-7.5 \mathrm{keV}\left({ }^{55} \mathrm{Fe} 5.95 \mathrm{keV}\right)$, center panel at $10-28 \mathrm{keV}\left({ }^{237} \mathrm{~Np}\right.$ lines: $11.89,13.9,17.81$ and $20.82 \mathrm{keV}$; ${ }^{241} \mathrm{Am}$ $26.34 \mathrm{keV})$ and right panel $54-61 \mathrm{keV}\left({ }^{241} \mathrm{Am} 59.6 \mathrm{keV}\right)$. The dark areas on these images are a shadow from the cover with beryllium entrance window of $30 \mathrm{~mm}$ diameter. The detector cover is transparent for the photons at energies higher than $50 \mathrm{keV}$, which is clearly seen on the right panel of this figure. 

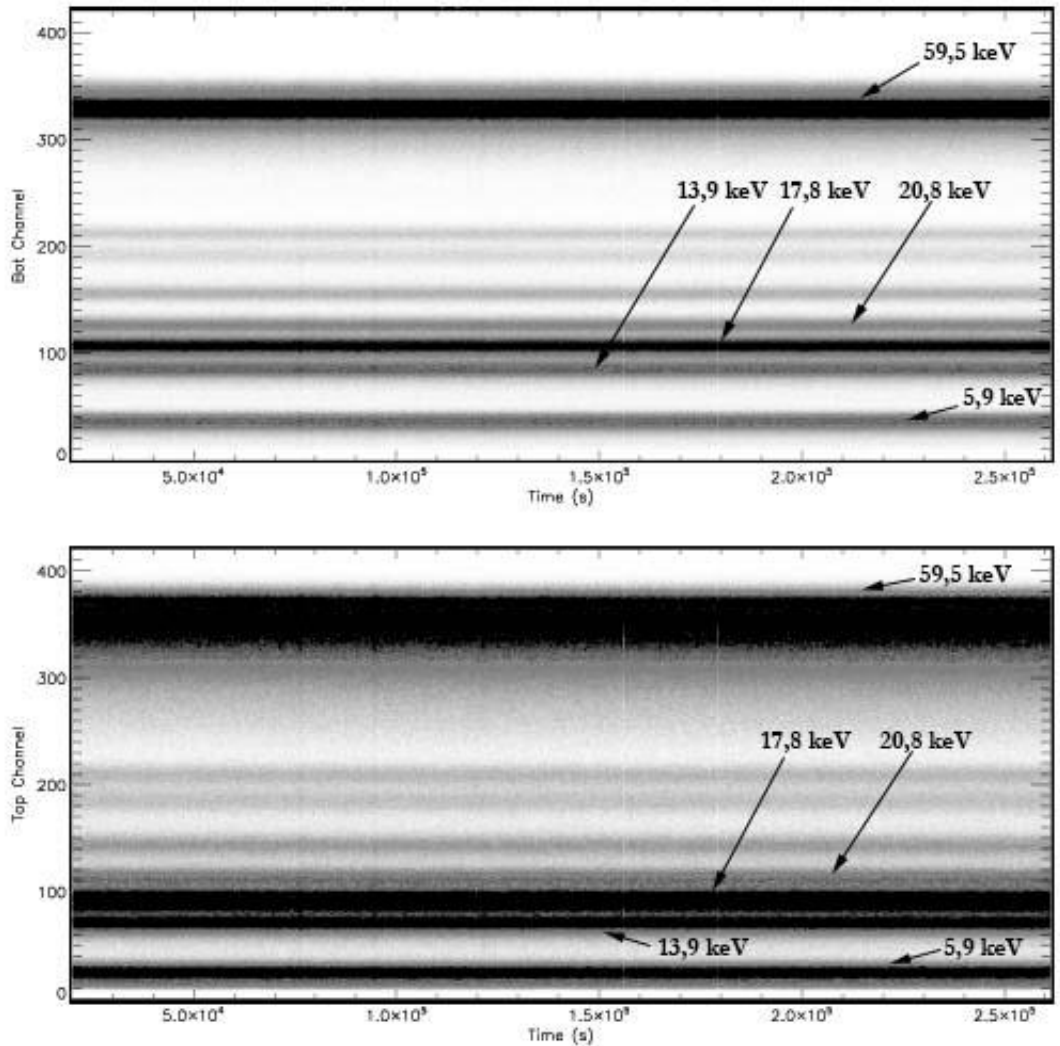

Figure 8. ${ }^{55} \mathrm{Fe}$ and ${ }^{241} \mathrm{Am}$ spectra as function of time for bottom (top panel) and top (bottom panel) layers of a QM detector. Every vertical line is the sum of spectra obtained in all 48 strips of each layer. Both spectra were stable up to 67 hours at $-29^{\circ} \mathrm{C}$ and $-100 \mathrm{~V}$ bias. 

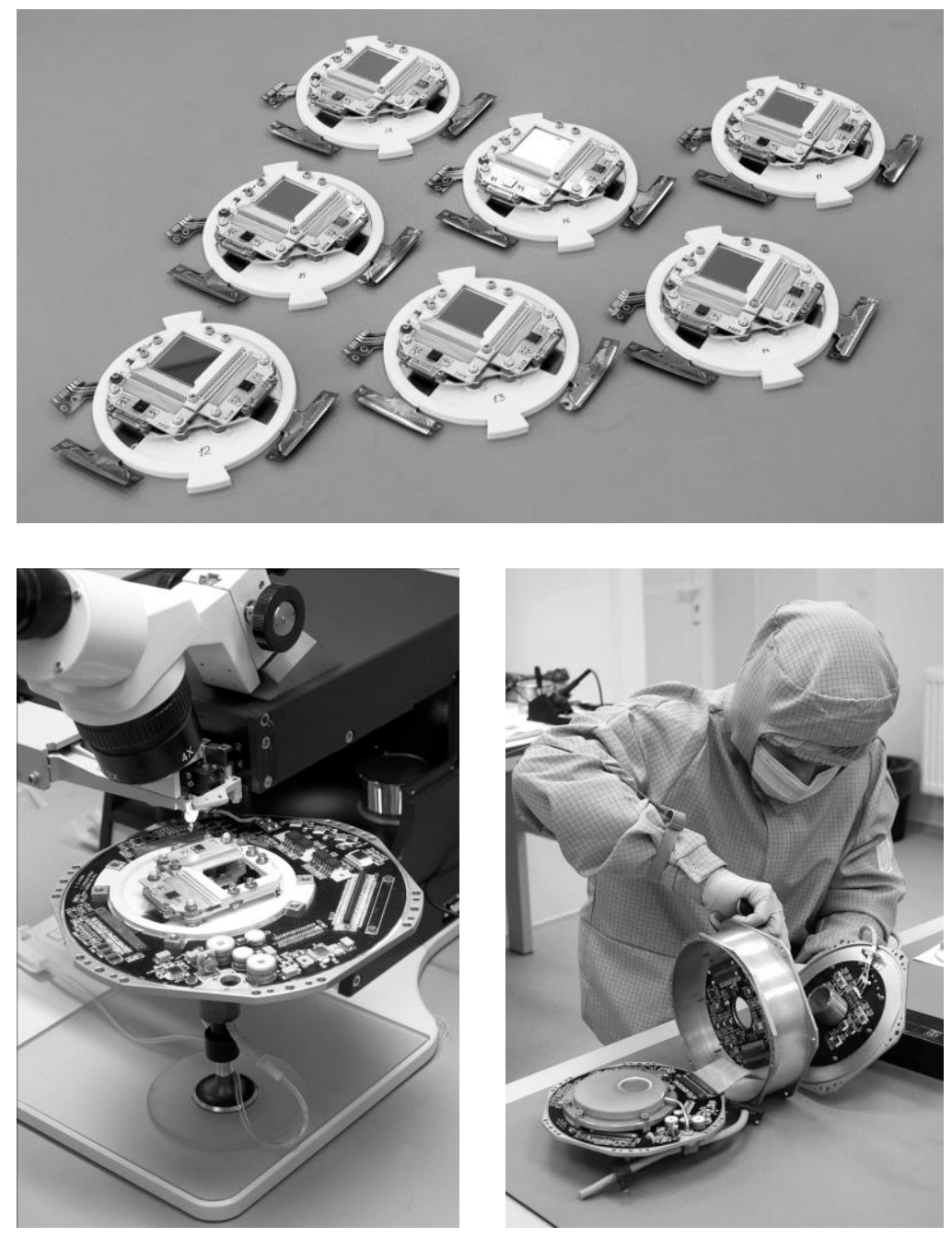

| Figure 9. The top photo shows seven qualification--model (QM) DSSD CdTe crystals and their holders ready to assemble. The bottom images show the assembly process of the QM ART-XC X-ray detector blocks. 


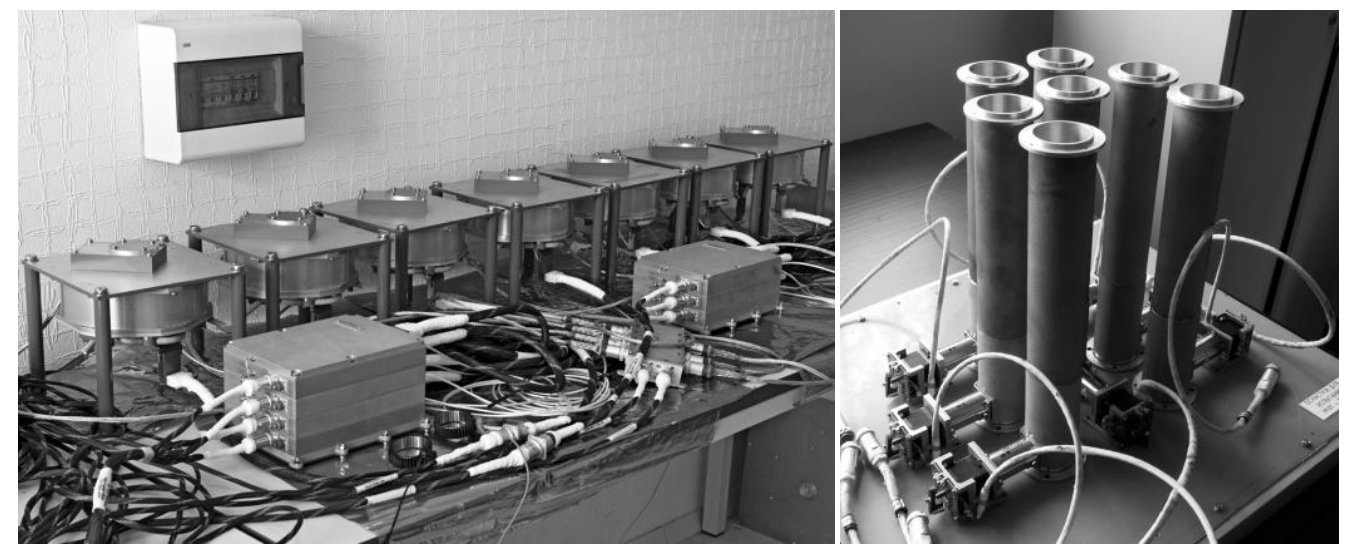

Figure 10. Left panel shows the QM ART-XC x-ray detector blocks [modules or systems] during a technological run at room temperature. Right panel shows the seven OM ART-XC collimators with blocks of the calibration source unitss.

Table 4. The ART-XC detector parameters

\begin{tabular}{|l|c|}
\hline Detector type & $\begin{array}{c}\text { CdTe Schottky Diode double sided strip } \\
\text { (ACRORAD, Japan) }\end{array}$ \\
\hline Size of CdTe crystal & $29.953 \times 29.953 \times 1 \mathrm{~mm}$ \\
\hline Working area & $28.56 \times 28.56 \mathrm{~mm}$ \\
\hline Strip width & $520 \mu \mathrm{m}$ \\
\hline Inter-strip distance & $75 \mu \mathrm{m}$ \\
\hline Number of strips & $48 \times 48$ \\
\hline ASIC & VA64TA1 \\
\hline Energy range & $5-80 \mathrm{keV}$ \\
\hline Energy resolution & $10 \%$ at $14 \mathrm{keV}$ \\
\hline Operating temperature & $-30^{\circ} \mathrm{C}$ \\
\hline Operating voltage & $-100 \mathrm{~V}$ \\
\hline Be entrance window diameter & $30 \mathrm{~mm}$ \\
\hline Be entrance window thickness & $100 \mu \mathrm{m}$ \\
\hline
\end{tabular}

\section{Science with ART-XC}

The scientific goals of the SRG mission are to study the large-scale structure of the Universe and explore the evolution of supermassive black holes. To achieve these goals SRG will survey the sky in the low and mid-energy band with unprecedented sensitivity, discovering a very large number of galaxy clusters and AGN.

ART-XC's role is to extend the energy range of the eROSITA instrument, significantly enhancing the sensitivity of the SRG mission at and above the critical iron-K region and facilitating the $\mathrm{X}$-ray detection of heavily obscured cosmic sources, especially local Active Galactic Nuclei.

As mentioned above, during the all-sky survey SRG will observe the whole sky every half year due to the Earth's rotation around the Sun. For downloading scientific data to the Earth the spacecraft is equipped with the fixed mediumgain antenna. In order to have the continuous downlink capability the spacecraft rotation axis will always be pointed between the Sun and the Earth so that the Earth will always be within the beam pattern of the medium gain antenna. Movement of the spacecraft around L2 point combined with the Earth's rotation around the Sun leads to nonuniform sky 
coverage. (see Figure 11). The final 3 years of mission will be spent on pointed observations of selected objects from the most interesting objects found. Therefore the SRG mission will effectively perform three surveys - the all sky survey, a concurrent order-of-magnitude deeper survey near the ecliptic poles and an even deeper 'blank-field' survey during long pointed observations (see Figure 12).

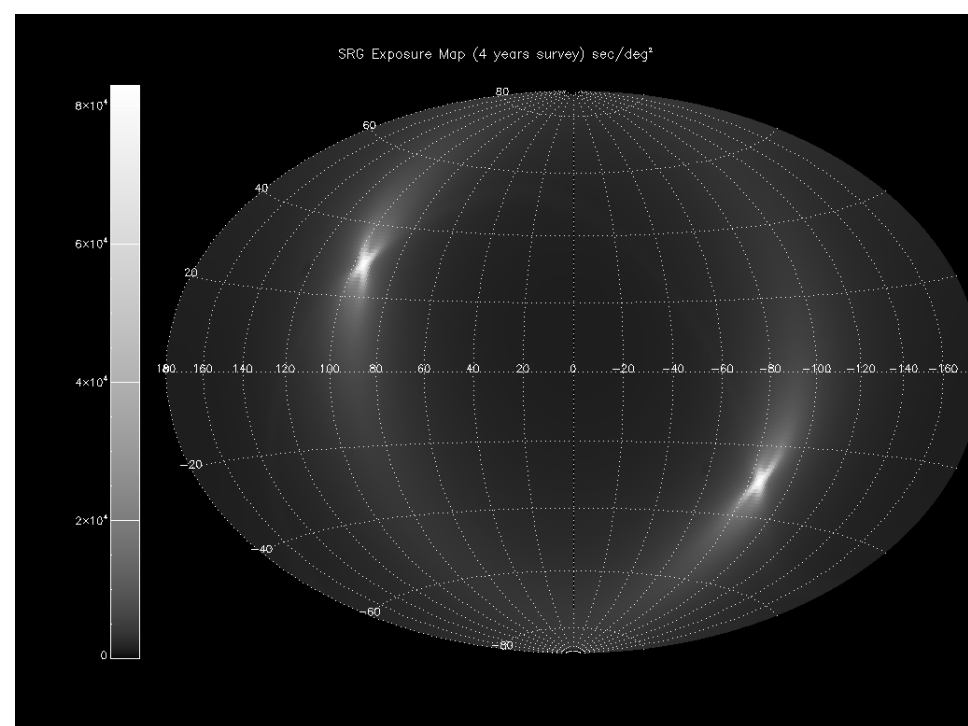

Figure 11. Exposure map (Galactic coordinates) for the 4-year survey of SRG in units of seconds/ square degree. The spacecraft rotation axis is always pointed between the Sun and the Earth to ensure that the Earth is always within the beam pattern of the spacecraft medium gain antenna. Movement of the spacecraft around the L2 point combined with the movement of the Earth around the Sun leads to nonuniform sky coverage.

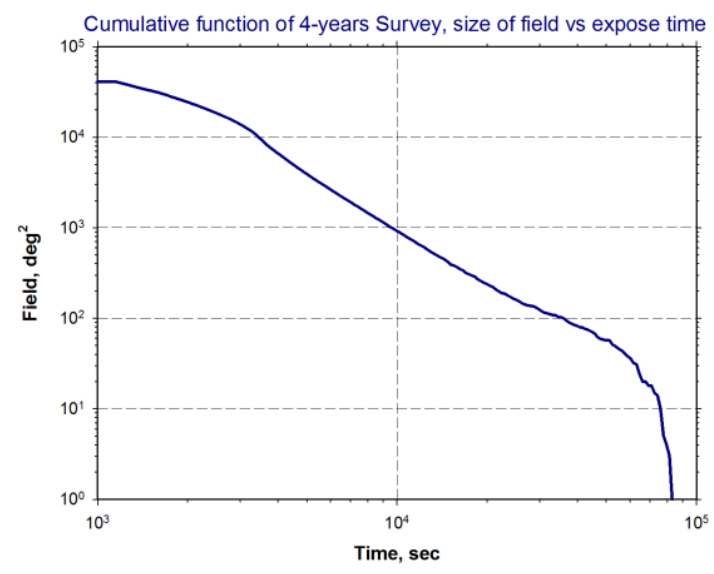

Figure 12. Cumulative function of the SRG 4 years survey. 
The harder response of ART-XC relative to eROSITA also facilitates the x-ray detection of obscured AGN. The combination of eROSITA + ART-XC enables the detection of some hundreds of heavily obscured AGN, a key population that accounts for over half of all AGN.

Finally, during the mission's pointing phase, the ART-XC instrument will study the spectra of heavily obscured galactic $\mathrm{X}$-ray binary systems and will study the broad-band spectra of various Galactic objects including binary systems, anomalous pulsars and supernova remnants up to $30 \mathrm{keV}$ with sufficient good spectroscopy and timing. Further, ART$\mathrm{XC}$ can study the non-thermal component in the galactic diffuse emission and perform searches for cyclotron line features in the spectra of X-ray pulsars.

\section{REFERENCES}

[1] Predehl, P., et al., "eROSITA on SRG", Proc. SPIE 7732, 77320U (2010).

[2] Pavlinsky, M., et al., "The ART-XC Instrument on board the SRG Mission", Proc. SPIE 8147, 814706-8147066 (2011). 